\title{
Digital photoelasticity for calculating coefficients of the Williams series expansion in plate with two collinear cracks under mixed mode loading
}

\author{
L.V. Stepanova ${ }^{1}$, V.S. Dolgikh ${ }^{1}$, V.A. Turkova ${ }^{1}$ \\ ${ }^{1}$ Samara National Research University, 34 Moskovskoe Shosse, 443086, Samara, Russia
}

\begin{abstract}
Photoelasticity method is used to study experimentally the complete Williams series expansion of the stress and displacement fields in the vicinity of the crack tip in isotropic linear elastic plates under Mixed Mode loading. The distribution of the isochromatic fridge patterns is employed for obtaining the stress field near the crack tip by the use of the complete Williams asymptotic expansion for various classes of the experimental specimens (plates with two collinear cracks under tensile loading and under mixed mode loading conditions). The higher order terms of the Williams series expansion are taken into account and the coefficients of the higher order terms are experimentally obtained. The stress field equation of Williams up to fifty terms in each in mode I and mode II has been considered. The comparison of the experimental results and the calculations performed with finite element analysis has shown the importance and significant advantages of photoelastic observations for the multi-parameter description of the stress field in the neighborhood of the crack tip.
\end{abstract}

Keywords: photoelastic stress analysis; elastic behavior; fracture; coefficients of the higher order terms

\section{Introduction}

The advent of computers coupled with developments in personal computer digital image processing has had a great influence in the development of modern photoelasticity [1-8]. The term "digital photoelasticity" refers to the automation of the photoelastic data collection and analysis [1]. The classical manual procedure of analysis is usually very tedious and time consuming and requires skilled and experienced personnel. With the advent of digital image processing techniques in photomechanics, digital photoelasticity has become very popular [1-9]. The development of the computer-aided analysis of the experimental data obtained from photoelastic experiment caused rapid growth of the photoelasticity experiments [1-20], especially in fracture mechanics for analysis of the crack tip fields and crack tip parameters. In the past two decades crack-tip mechanics has been studied increasingly using full-field techniques, namely DIC [15] and photoelasticity [1-8].

Thus, in [2] the experimental technique of photoelasticity has been utilized for calculating bi-material notch stress intensities as well as the coefficients of higher order terms. Employing the equations of multi-parameter stress field allows data collection from a larger zone from the notch tip and makes the data collection from experiments more convenient. Moreover, the effects of higher order terms in the region near the notch tip are taken into account. For the photoelasticity experiments, a laboratory specimen known as the Brazilian disk with a central notch, consisting of aluminum and polycarbonate, has been utilized in [2]. Using this specimen, different mode mixities could be easily produced by changing the loading angle. The bi-material notch stress intensities and the first non-singular stress term (called T-stress) were calculated for different test configurations. In order to utilize the advantages of whole-field photoelasticity and minimize the experimental errors, a large number of data points were substituted in the multiparameter stress field equations. Then the resulting system of nonlinear equations was solved by employing an over-deterministic least squares method coupled with the Newton-Raphson algorithm. It has been shown [2] that considering the T-stress term improves, to a large extent, the accuracy of the stress intensities calculated through the photoelasticity technique. Moreover, by reconstructing the isochromatic fringes, the effects of the T-stress term on the shape and size of these fringes around the notch tip were investigated for a 30 notch. The experimental photoelasticity results were also compared with the corresponding values obtained from finite element analysis and a good correlation was observed.

In [3] it is noted that the V-notches are most possible case for initiation of cracks in structure elements. The specifications of cracks on the tip of the notch will be influenced via opening angle, tip radius and depth of V-notch. In [3] the effects of Vnotch's opening angle on stress intensity factor and T-stress of crack on the notch has been investigated. The experiment has been done in different opening angles and various crack length in mode I loading using photoelasticity method. The results illustrate that while angle increases in constant crack's length, stress intensity factor (SIF) and T-stress will decrease. Beside, the effect of V-notch angle in short crack is more than long crack. These V-notch affects are negligible by increasing the length of crack, and the crack's behavior can be considered as a single-edge crack specimen.

Guaglianon [4] et al considered the multi-parameter description of the crack tip in isotropic linear elastic materials. The elastic stress field around a crack tip is fully defined through multiparameter equations. A code and program were implemented to evaluate the characteristic parameters of the stress field around a crack tip by photoelastic analysis. The possibility to change the number of parameters makes it possible to adapt the study to different cases, increasing the extension of the analyzed area in order to have a correct modeling of the photoelastic fringes. The performed experimental tests allow emphasizing the importance of using multiparameter equations in the study of the stress field around the crack tip.

Inspired by the Brazilian disk geometry the authors of [5] examine the utility of an edge cracked semicircular disk (ECSD) specimen for rapid assessment of fracture toughness of brittle materials using compressive loading. It is desirable to optimize the geometry towards a constant form factor for evaluating SIF $K_{I}$. In this investigation photoelastic and finite element results for $K_{I}$ evaluation highlight the effect of loading modeled using a Hertzian loading. According to authors of [5] a Hertzian loading subtending 4 at the center leads to a surprisingly constant form factor of 1.36. This special case is further analyzed by applying uniform pressure over a chord for facilitating testing.

$3^{\text {rd }}$ International conference "Information Technology and Nanotechnology 2017" 
Lei et al [6] applied photoelasticity method for study of structural imperfection of $\mathrm{ZnGeP}_{2}$ crystal. The stresses related to rows and accumulations of dislocations were revealed by photoelastic method for $\mathrm{ZnGeP}_{2}$ crystals grown by Vertical Bridgman method. A comparison of information from topographs of photoelastic method and X-Ray topography based on Borrmann method was carried out. It was shown that the strongest contrast is observed on boundaries of dislocation rows and regions of relatively perfect crystals. Photoelastic method gives information about defect structure where X-Ray topography can not be applied because of high density of defect sand disorientation of reflection planes. Because of high sensitivity of photoelastic method the images of defects have larger size then in X-Ray topography. That is why in $\mathrm{ZnGeP}_{2}$ predominately the total contrast from dislocation rows is fixed. However, in low angle boundaries photoelastic images of separate dislocations were revealed. By comparison with results of simulation it was stated that they are created by edge dislocation of slip system $\{110\}(110)$ what confirms the data obtained by Borrmann method. Thus, photoelastic method can be, from one side, a simple and express method of analysis of $\mathrm{ZnGeP}_{2}$ plates cut along the plane of optical isotropy (001) and, from other side, an analytical method of identification of dislocations and other defects in this material.

In [7] it is noted once again that the photoelastic technique has seen some renewed interest in past few years with digital images and image processing new methods becoming readily available. However, further research is needed to improve the precision, the accuracy and the automation of photoelastic technique. The aim of [7] is to get new numerical equations for the phase-shifting method in digital photoelasticity using a plane polariscope. The model was developed to plane polariscope because of the simplicity and low cost of this equipment. To develop the phase shift and respective intensity equations only the analyzer is rotated. A ring under diametral compression is used for the experimental validation. From these intensity equations the equations for isoclinic and isochromatic parameters are deduced by applying a new numerical technique. This approach can be used to calculate the isoclinic and isochromatic parameters using any number of images. Several analyses are performed with different number of photographic images. The results showed errors reduce when more phase-stepped images are utilized. Hence, one concludes that the uncertainties in results due to effects of errors on photoelastic images can be reduced with a larger amount of phase-stepped images.

In [8] the recent advances in digital photoelasticity have made it possible to use it conveniently for the stress analysis of articles and components made of glass. Depending on the application the retardation levels to be measured range from a few nanometres to several thousand nanometres, which necessitates different techniques and associated equipments. This paper [8] reviews the recent advances in the photoelasticity of glass with a focus on the techniques/methods developed in the last decade.

The aim of the present study is to found coefficients of the multiparameter asymptotic expansion of the stress field in the vicinity of the tips of two collinear cracks in the isotropic linear elastic plate using the photoelasticity method. The main idea of the paper is to keep the higher-order terms of the Williams series expansion of the stress filed, to reveal and evaluate the effect of the higher-order terms of the asymptotic expansion. The motivation of this study is twofold. First, we would like to compare theoretical results obtain in $[9,10]$ with experimental data. According to $[9,10]$ the higher-order terms of the complete asymptotic expansion of the crack-tip stress field can play a significant role. The more distance from the crack tip the more terms it is necessary to keep in the Williams asymptotic expansion. Thus it follows the second reason when the photoelastic data is processing the number of terms of the asymptotic expansion can't be chosen arbitrary. It depends on the distance from the crack tip. Therefore, the distance from the crack tip should be taken into account when we suppose the structure of the solution in the vicinity of the crack tip.

Note that the coefficients of higher-order terms in the Williams series expansions were computed by different approaches in numerous studies [11-15].

In [11] the digital photoelasticity technique is used to estimate the crack tip fracture parameters for different crack configurations. Conventionally, only isochromatic data surrounding the crack tip is used for SIF estimation, but with the advent of digital photoelasticity, pixel-wise availability of both isoclinic and isochromatic data could be exploited for SIF estimation in a novel way. A linear least square approach is proposed to estimate the mixed-mode crack tip fracture parameters by solving the multi-parameter stress field equation. The stress intensity factor (SIF) is extracted from those estimated fracture parameters. The isochromatic and isoclinic data around the crack tip is estimated using the ten step phase shifting technique. To get the unwrapped data, the adaptive quality guided phase unwrapping algorithm (AQGPU) has been used. The mixed mode fracture parameters, especially SIF are estimated for specimen configurations like single edge notch (SEN), center crack and straight crack ahead of inclusion using the proposed algorithm. The experimental SIF values estimated using the proposed method are compared with analytical/finite element analysis (FEA) results, and are found to be in good agreement.

In [12] the method of photoelasticity is used to study the effects of first non-singular stress term on isochromatic fringe patterns around the tip of a mode I sharp V-notch. Notches are divided into two categories: notches with opening angles a) less than 45 , and b) between two angles 45 and 152 . First, utilizing the mathematical relations of the isochromatic fringes, the effects of the first non-singular stress term on the shape and size of the fringes are studied theoretically. For notch opening angles less than 45 , it is shown that the isochromatic fringes rotate forward and backward when the coefficient of the first nonsingular term is negative and positive, respectively. It is also demonstrated that both backward and forward rotations of fringe patterns are possible when the notch angle is between 45 and 152 . For all notch opening angles, as the first non-singular term dominates the notch tip stress field, a new type of fringe appears far from the notch tip. In order to evaluate the analytical findings, a photoelastic test program is also performed on a centrally notched cruciform specimen. Using this specimen, different loading conditions are simulated by changing the lateral load ratio and consequently different effects of the first non-singular term on the shape and size of the fringes are investigated experimentally. Good correlation between the analytical and experimental results is observed.

Harilal et al [13] an experimental study is carried out to estimate the mixed-mode stress intensity factors (SIF) for different cracked specimen configurations using digital image correlation (DIC) technique. For the estimation of mixed-mode SIF's using 
DIC, a new algorithm is proposed for the extraction of crack tip location and coefficients in the multi-parameter displacement field equations. From those estimated coefficients, SIF could be extracted. The required displacement data surrounding the crack tip has been obtained using 2D-DIC technique. An open source 2D DIC software Ncorr is used for the displacement field extraction. The presented methodology has been used to extract mixed-mode SIF's for specimen configurations like single edge notch (SEN) specimen and centre slant crack (CSC) specimens made out of A12014-T6 alloy. The experimental results have been compared with the analytical values and they are found to be in good agreement, there by confirming the accuracy of the algorithm being proposed.

The coefficients of higher-order terms in the Williams series expansions were computed using the DIC method which is a noncontact full-field optical technique [15]. First, the fundamental concepts of DIC method were described and then, this method was proposed to obtain the higher-order terms of the Williams expansion for a CT specimen under pure mode I loading. The displacement field around the crack tip in the CT specimen was determined by the DIC approach. The displacements were utilized in order to obtain the coefficients of Williams expansion. Then, these coefficients were also calculated by using the FE method from the displacement field in the vicinity of the crack tip. The values of stress intensity factor and T-stress obtained from the DIC and FE techniques were compared with the results of previous researches. The efficiency and accuracy of the DIC technique in determining the coefficients of higher order terms in the Williams expansion were demonstrated for the CT specimen. As it is noted in [11] the accuracy of SIF estimate could be improved by improving the accuracy of the isoclinic parameter estimate using the white light photoelasticity thereby eliminating the isochromatic-isoclinic interaction noise. An advanced experimental technique for determination of the stress intensity factor (SIF) and the T-stress is developed in [18] and carefully verified. The approach employs optical interferometric measurements of local deformation response to small crack length increment. Narrow notches are used for crack modeling. Initial experimental data represent inplane displacement component values measured by electronic speckle-pattern interferometry in the vicinity of the crack tip. Determination of the first four coefficients of Williams' series is the main feature of the developed technique. Relationships for transition from measured in-plane displacement components to required fracture mechanics parameters are presented. Availability of highquality interference fringe patterns, which are free from rigid-body motion, serves as a reliable indicator of real strain state near the crack tip. Experimental verification of the proposed method is performed for non-symmetrical and symmetrical crack in thin rectangular plates subjected to uniaxial tension. The distributions of SIF and T-stress values for cracks of different length in residual stress fields near electronically welded joints of thin plates are presented as an example of practical implementing.

In [19] the common definitions for mode I and mode II are evaluated and improved. For this purpose, the in-plane linear elastic stress field around the crack tip is written as a set of infinite series expansions. Mode I and mode II fields are classically defined as symmetric and anti-symmetric parts of these expansions, respectively. There is also a constant term called "T-stress" in these expansions; parallel to the crack line and independent of the distance from the crack tip. Previous definitions assume that Ts-tress exists only in pure mode I or combined mode I and mode II conditions. Based on these definitions, T-stress always vanishes in pure mode II. However, the published results of several analytical and experimental researches indicate that the constant stress term can exist in mode II stress field, as well. In this paper, some examples are presented which indicate the presence and importance of T-stress in pure mode II conditions. Then, the classical definition for mode I and mode II is modified to make it consistent with the results presented in the literature.

Thus, the photoelasticity techniques have been extensively used for experimentally determining the state of stress in actual mechanical components. In this research, photoelasticity was employed to assess the singular and higher-order coefficients of the Williams series expansion for the stress field in the vicinity of the crack tip. To utilize the advantages of the whole - field photoelasticity and minimize the experimental errors, the overdeterministic method [18] has been used. The experimental equipment is shown in Fig. 1. The aim of this paper is to obtain the coefficients of the higher-order terms in the Williams expansion and to estimate the influence of these terms on the stress field description taking into account as many as possible terms in the asymptotic presentation of the crack tip fields.

\section{Elastic stress field around the crack tip}

In the development of linear fracture mechanics M. Williams made a major breakthrough in the analysis of the asymptotic stress field at the vicinity of the crack tip in isotropic linear elastic plane media. With the eigenfunction expansion method it is possible to establish the separable variable nature of the solution and to obtain asymptotic expressions for the stress field in a plane medium with a traction-free crack submitted to mode I, mode II and mixed-mode (mode I and mode II) loading conditions:

$$
\sigma_{i j}(r, \theta)=\sum_{m=1}^{2} \sum_{k=-\infty}^{\infty} a_{k}^{m} r^{k / 2-1} f_{m, i j}^{(k)}(\theta)
$$

with index $m$ associated to the fracture mode; $a_{k}^{m}$ amplitude coefficients related to the geometric configuration, load and mode; $f_{m i, j}^{(k)}(\theta)$ angular functions depending on stress component and mode loadings. Analytical expressions for angular eigenfunctions $f_{m, i j}^{(k)}(\theta)$ are available $[9,16]$ :

$$
\begin{aligned}
& f_{1,11}^{(k)}(\theta)=\frac{k}{2}\left[\left(2+k / 2+(-1)^{k}\right) \cos (k / 2-1) \theta-(k / 2-1) \cos (k / 2-3) \theta\right], \\
& f_{1,22}^{(k)}(\theta)=\frac{k}{2}\left[\left(2-k / 2-(-1)^{k}\right) \cos (k / 2-1) \theta+(k / 2-1) \cos (k / 2-3) \theta\right], \\
& f_{1,12}^{(k)}(\theta)=\frac{k}{2}\left[-\left(k / 2+(-1)^{k}\right) \sin (k / 2-1) \theta+(k / 2-1) \sin (k / 2-3) \theta\right],
\end{aligned}
$$

$3^{\text {rd }}$ International conference "Information Technology and Nanotechnology 2017" 


$$
\begin{aligned}
& f_{2,11}^{(k)}(\theta)=-\frac{k}{2}\left[\left(2+k / 2-(-1)^{k}\right) \sin (k / 2-1) \theta-(k / 2-1) \sin (k / 2-3) \theta\right], \\
& f_{2,22}^{(k)}(\theta)=-\frac{k}{2}\left[\left(2-k / 2+(-1)^{k}\right) \sin (k / 2-1) \theta+(k / 2-1) \sin (k / 2-3) \theta\right], \\
& f_{2,12}^{(k)}(\theta)=\frac{k}{2}\left[-\left(k / 2-(-1)^{k}\right) \cos (k / 2-1) \theta+(k / 2-1) \cos (k / 2-3) \theta\right] .
\end{aligned}
$$

Characteristics of the fracture problems such as the geometry of the specimen and intensity of the load influence neither radial nor angular functions in equation (1) [21]. All the variety of fracture mechanics problems is therefore taken into account in the sole sequence of coefficients $a_{k}^{m}$.Terms with higher orders have been proven to be influential in some circumstances [9,10,16, 21-34]. In the present paper the experimental technique of photoelasticity was employed to investigate the effects of the higher order terms on both the shape and the size of the near crack tip isochromatic fringes for the wide range of specimens.

\section{Photoelastic determination of coefficients of the higher order terms in the Williams series expansion}

Based on the classical concepts of photoelasticity, the locus of an isochromatic fringe is expressed by the stress optic law

$$
2 \tau_{m}=\frac{N f_{\sigma}}{h}
$$

where $\tau_{m}$ is the maximum in-plane shear stress, $N$ and $f_{\sigma}$ represent the fringe order and the material stress-fringe value, respectively, $h$ is the specimen thickness. On the other hand, the relation between the maximum shear stress $\tau_{m}$ and the Cartesian stress components is

$$
\tau_{m}^{2}=\left(\sigma_{11}-\sigma_{22}\right)^{2} / 4+\sigma_{12}^{2} .
$$

The maximum in-plane shear stress around the crack tip can be described by considering $K$ terms of Mode I and $M$ terms of Mode II expansion from equation (1). By substituting the truncated series expansion of equation (1) (with $K$ terms of Mode I and $M$ terms of Mode II) and equation 5 into equation 4 the mathematical equation for a fringe developed around the crack tip can be written as

$$
\begin{aligned}
& \left(\frac{N f}{2 h}\right)^{2}=\left(\sigma_{11}-\sigma_{22}\right)^{2} / 4+\sigma_{12}^{2}=\left(\sum_{k=1}^{K} a_{k}^{1} r^{k / 2-1} f_{1,11}^{(k)}(\theta)+\sum_{k=1}^{M} a_{k}^{2} r^{k / 2-1} f_{2,11}^{(k)}(\theta)-\sum_{k=1}^{K} a_{k}^{1} r^{k / 2-1} f_{1,22}^{(k)}(\theta)-\sum_{k=1}^{K} a_{k}^{2} r^{k / 2-1} f_{2,22}^{(k)}(\theta)\right)^{2} / 4+ \\
& +\left(\sum_{k=1}^{K} a_{k}^{1} r^{k / 2-1} f_{1,12}^{(k)}(\theta)+\sum_{k=1}^{M} a_{k}^{2} r^{k / 2-1} f_{2,12}^{(k)}(\theta)\right)^{2}
\end{aligned}
$$

Equation (6) provides $K$ and $M$ terms in Mode I and Mode II expansions, respectively to represent the near crack tip stress field. Further the method of evaluation of mixed-mode stress field parameters proposed in [17] is used. The method is called as an over-deterministic method for calculating the stress intensity factor as well as the coefficients of the higher-order terms in the Williams series expansions in cracked bodies.

\subsection{Experimental procedure}

A set of cracked specimens was used to calculate the stress intensity factor and the coefficients of the higher-order terms of the complete Williams series expansion for the stress field in the vicinity of the crack tip. These specimens are the plate with two collinear crack of the equal length (Fig. 3), the plates with two collinear cracks of different lengths (Fig. 4), the plates with two inclined collinear cracks of the equal and different lengths (Fig. 5).

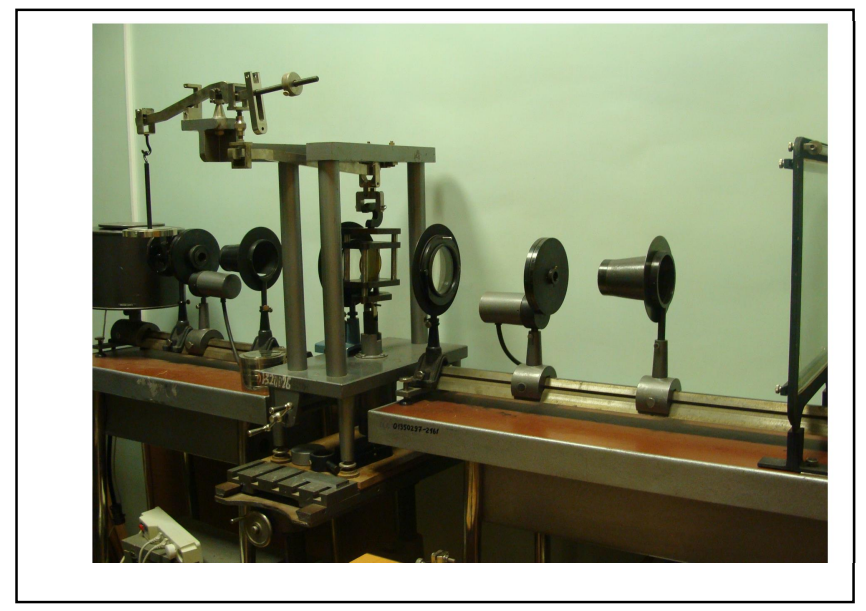

Fig. 1. Experimental setup. 
The value of the fringe constant is determined experimentally by inducing a known stress difference $\sigma_{1}-\sigma_{2}$ in a model that is made of the same material as the specimen of interest by observing the corresponding value of $N$ and by solving the optic law $\sigma_{1}-\sigma_{2}=N f_{\sigma} / h$ for $f_{\sigma}$. A common calibration specimen is circular disk (Fig. 2). The isochromatic fringe patter of circular disk under diametral compression is shown in Fig. 1 . The material fringe constant is $f_{\sigma}=18.33 \mathrm{~Pa} \mathrm{~cm} /$ fringe.

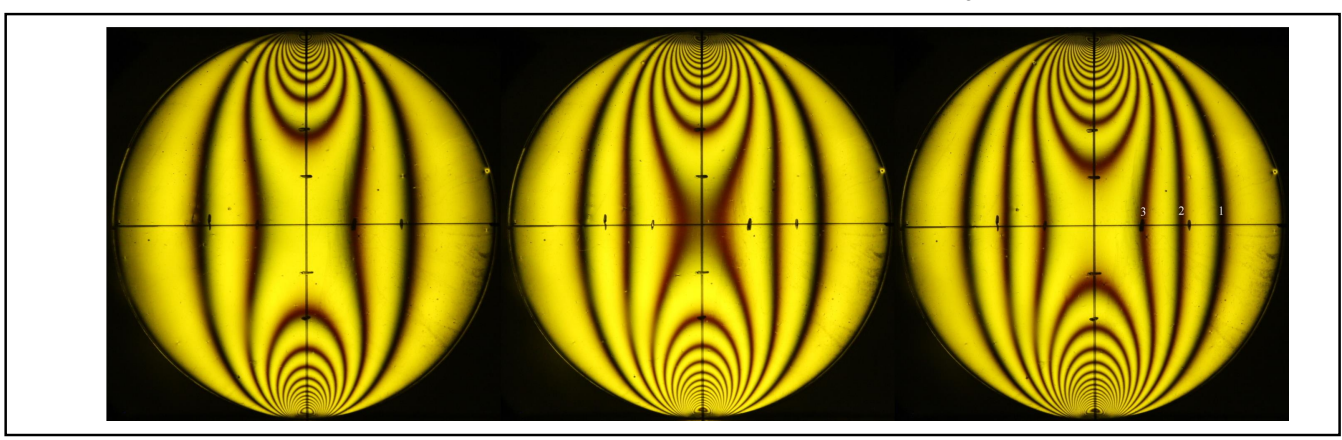

Fig. 2. Photoelastic yellow-light isochromatics for a disk in diametrical compression by load $14 \mathrm{~N}$ (left), $18 \mathrm{~N}$ (center) and $21 \mathrm{~N}$ (right).

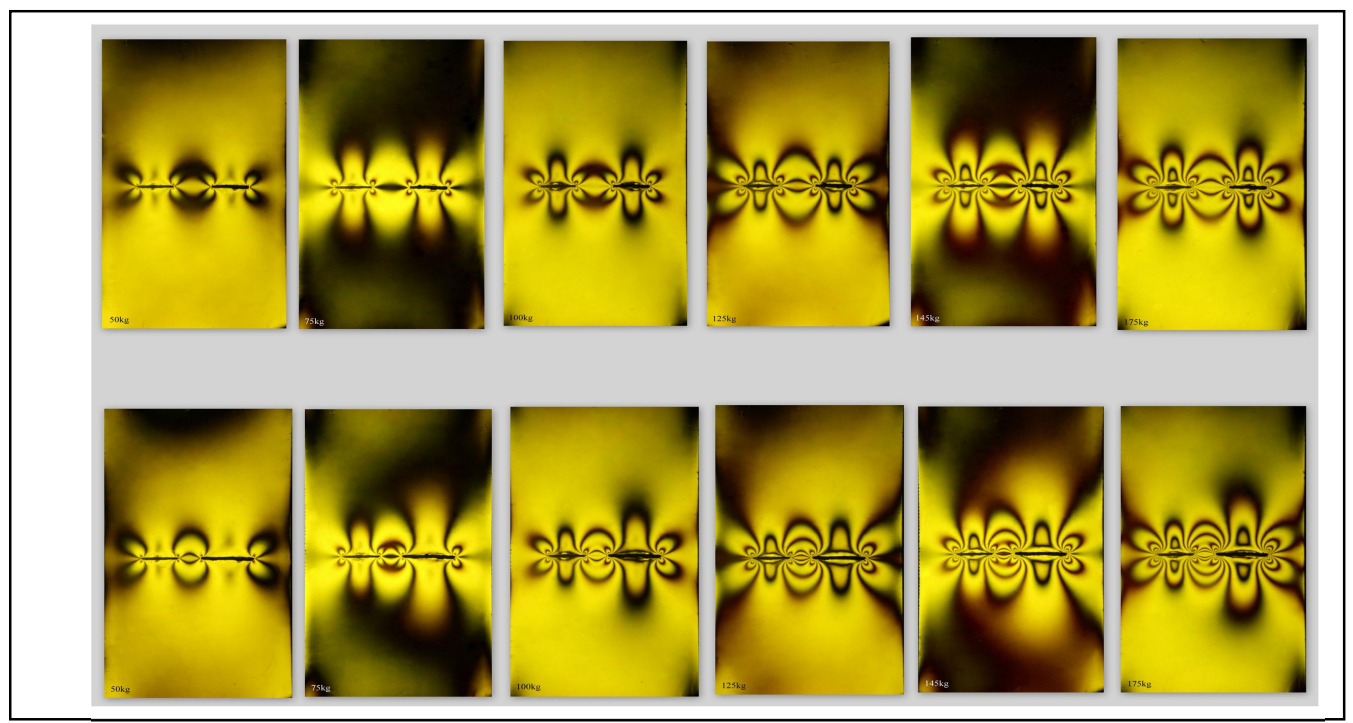

Fig. 3. Fhotoelastic isochromatic fringe patterns for plates with two collinear cracks under different loads.

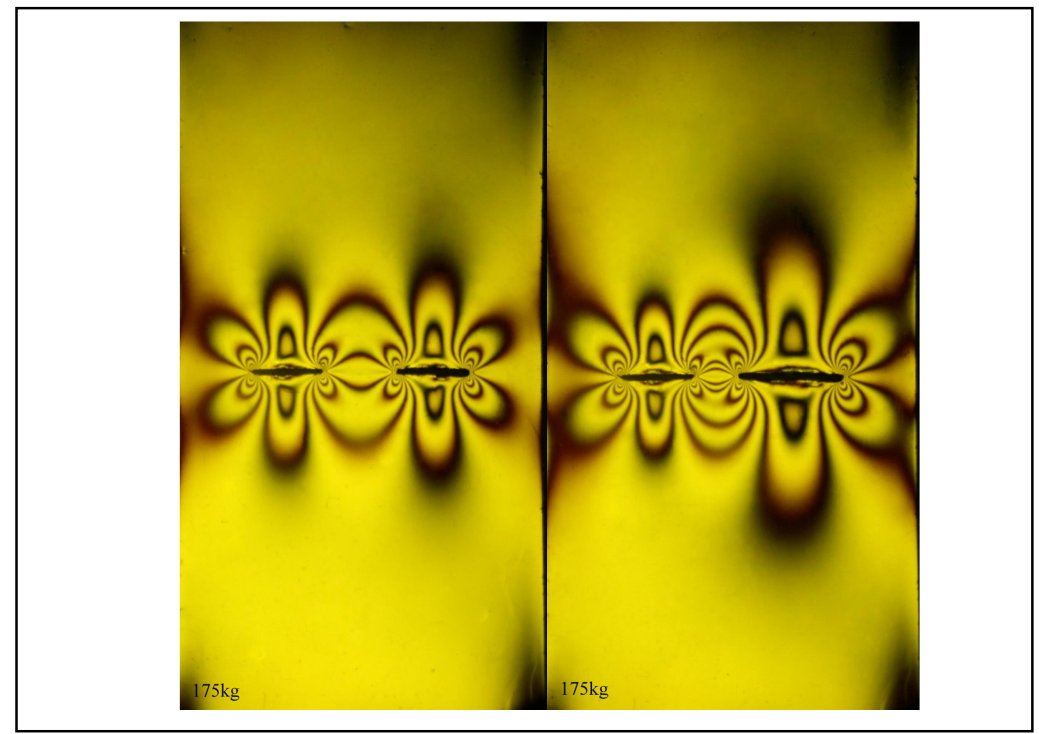

Fig. 4. Isochromatic fringes for a plate with two collinear cracks of different lengths.

After the image acquisition several interactive programs were developed for determining coefficients of higher-order terms. The necessary photoelastic data for a large number of selected points were collected using MATLAB. The algorithm is based on the fact that each pixel of a grayscale photograph has a value or intensity in the range of $0-255$, such that a pixel of 0.0 is displayed as black, a pixel value of 255 is displayed as white. Hence, pixels with lower intensities are corresponding to the darker points of grayscale photos. The computer code as it was proposed by M.R. Ayatollahi and M. Nejati in [14] was also developed. The code allows us to find the points of isochromatic fringe pattern. The approach was repeated for several lines in 
different directions and the darkest point of every isochromatic fringe in each radial direction were found. The positions of these points were collected and used. The collection of these points is used for determination of the higher order terms of the Williams asymptotic expansion for the stress field in the vicinity of the crack tip. The number of the points depends on the isochromatic fringe. The schematic presentation of the specimen geometry is shown in Fig. 6 . The normalized coefficients $a_{k}^{1}=a_{k}^{1} / \sigma_{22}^{\infty}$ are obtained for different types of specimens and presented in Tables 1-5.

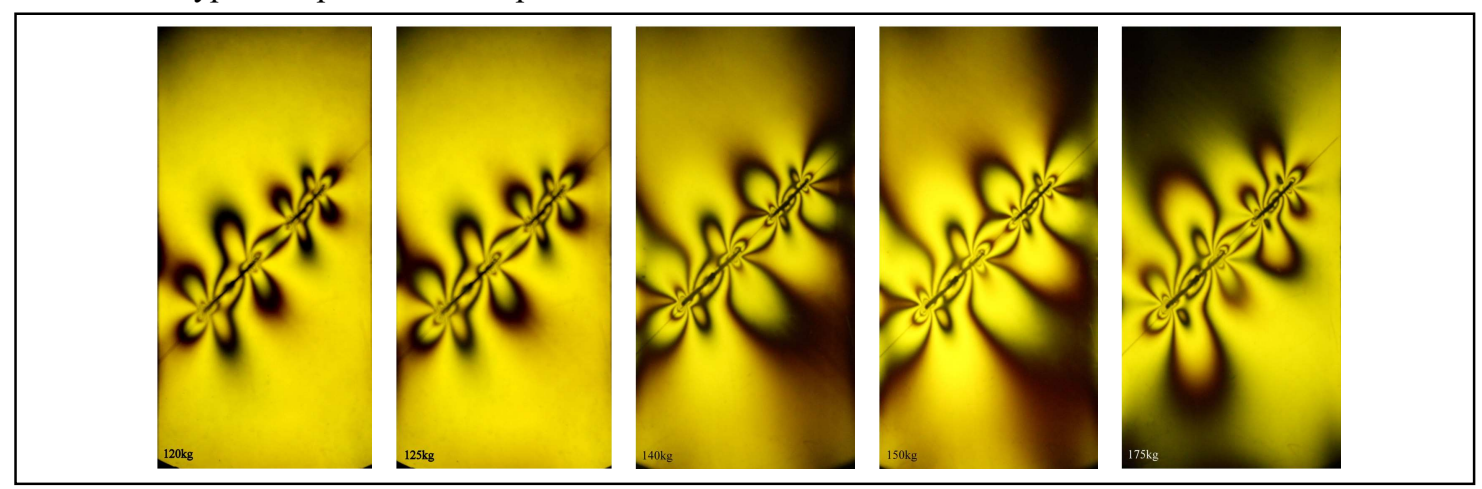

Fig. 5. Isochromatica fringe patterns in plates with two inclined collinear cracks under differents loads.

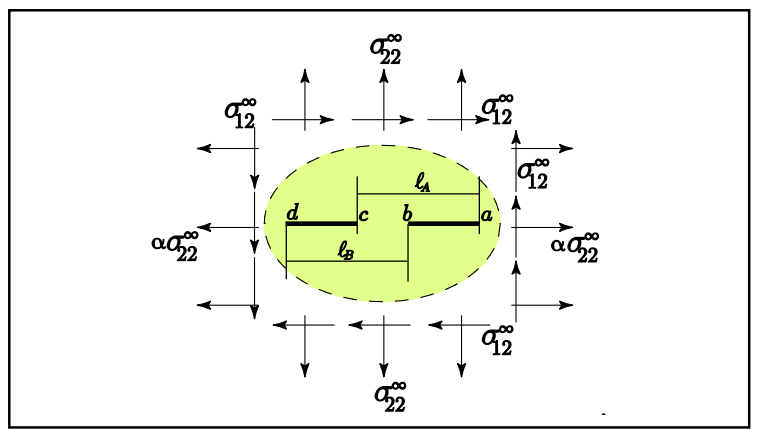

Fig. 6. Schematical presentaion of the specimen geometry considered.

Table 1. The coefficients of the Williams series expansion for the stress field in the vicinity of the crack tip $z=a$

$(a=1.5 \mathrm{~cm}, b=0.5 \mathrm{~cm}, c=-0.5 \mathrm{~cm}, d=-1.5 \mathrm{~cm})$.

\begin{tabular}{|c|c|c|c|c|c|c|c|c|}
\hline$a_{1}^{1}\left(\mathrm{~cm}^{-3 / 2}\right)$ & $a_{2}^{1}\left(\mathrm{~cm}^{-2}\right)$ & $a_{3}^{1}\left(\mathrm{~cm}^{-5 / 2}\right)$ & $a_{5}^{1}\left(\mathrm{~cm}^{-7 / 2}\right)$ & $a_{7}^{1}\left(\mathrm{~cm}^{-9 / 2}\right)$ & $a_{9}^{1}\left(\mathrm{~cm}^{-11 / 2}\right)$ & $a_{11}^{1}\left(\mathrm{~cm}^{-13 / 2}\right)$ & $a_{13}^{1}\left(\mathrm{~cm}^{-15 / 2}\right)$ & $a_{15}^{1}\left(\mathrm{~cm}^{-17 / 2}\right)$ \\
\hline $\begin{array}{l}0.5139 \\
a_{17}^{1}\left(\mathrm{~cm}^{-19 / 2}\right)\end{array}$ & $\begin{array}{l}-0.2500 \\
a_{19}^{1}\left(\mathrm{~cm}^{-21 / 2}\right)\end{array}$ & $\begin{array}{l}0.2512 \\
a_{21}^{1}\left(\mathrm{~cm}^{-23 / 2}\right)\end{array}$ & $\begin{array}{l}-0.0646 \\
a_{23}^{1}\left(\mathrm{~cm}^{-25 / 2}\right)\end{array}$ & $\begin{array}{l}0.0330 \\
a_{25}^{1}\left(\mathrm{~cm}^{-27 / 2}\right)\end{array}$ & $\begin{array}{l}-0.0208 \\
a_{27}^{1}\left(\mathrm{~cm}^{-29 / 2}\right)\end{array}$ & $\begin{array}{l}0.0145 \\
a_{29}^{1}\left(\mathrm{~cm}^{-31 / 2}\right)\end{array}$ & $\begin{array}{l}-0.0109 \\
a_{31}^{1}\left(\mathrm{~cm}^{-33 / 2}\right)\end{array}$ & $\begin{array}{l}0.0086 \\
a_{33}^{1}\left(\mathrm{~cm}^{-35 / 2}\right)\end{array}$ \\
\hline $\begin{array}{l}-0.0069 \\
a_{35}^{1}\left(\mathrm{~cm}^{-37 / 2}\right)\end{array}$ & $\begin{array}{l}0.0058 \\
a_{37}^{1}\left(\mathrm{~cm}^{-39 / 2}\right)\end{array}$ & $\begin{array}{l}-0.0049 \\
a_{39}^{1}\left(\mathrm{~cm}^{-41 / 2}\right)\end{array}$ & $\begin{array}{l}0.00426 \\
a_{41}^{1}\left(\mathrm{~cm}^{-43 / 2}\right)\end{array}$ & $\begin{array}{l}-0.0037 \\
a_{43}^{1}\left(\mathrm{~cm}^{-45 / 2}\right)\end{array}$ & $\begin{array}{l}0.0033 \\
a_{45}^{1}\left(\mathrm{~cm}^{-47 / 2}\right)\end{array}$ & $\begin{array}{l}-0.0029 \\
a_{47}^{1}\left(\mathrm{~cm}^{-49 / 2}\right)\end{array}$ & $\begin{array}{l}0.0026 \\
a_{49}^{1}\left(\mathrm{~cm}^{-51 / 2}\right)\end{array}$ & $\begin{array}{l}-0.0024 \\
a_{51}^{1}\left(\mathrm{~cm}^{-53 / 2}\right)\end{array}$ \\
\hline 0.0022 & -0.0020 & 0.0018 & -0.0017 & 0.0016 & -0.00015 & 0.0014 & -0.0013 & 0.0012 \\
\hline
\end{tabular}

Table 2. The coefficients of the Williams series expansion for the stress field in the vicinity of the crack tip $z=\tilde{n}$ (Fig. 6)

$(a=1.5 \mathrm{~cm}, b=0.5 \mathrm{~cm}, c=-0.5 \mathrm{~cm}, d=-1.5 \mathrm{~cm})$.

\begin{tabular}{lllllllll}
\hline$a_{1}^{1}\left(\mathrm{~cm}^{-3 / 2}\right)$ & $a_{2}^{1}\left(\mathrm{~cm}^{-2}\right)$ & $a_{3}^{1}\left(\mathrm{~cm}^{-5 / 2}\right)$ & $a_{5}^{1}\left(\mathrm{~cm}^{-7 / 2}\right)$ & $a_{7}^{1}\left(\mathrm{~cm}^{-9 / 2}\right)$ & $a_{9}^{1}\left(\mathrm{~cm}^{-11 / 2}\right)$ & $a_{11}^{1}\left(\mathrm{~cm}^{-13 / 2}\right)$ & $a_{13}^{1}\left(\mathrm{~cm}^{-15 / 2}\right)$ & $a_{15}^{1}\left(\mathrm{~cm}^{-17 / 2}\right)$ \\
\hline 0.52398 & -0.25000 & 0.27936 & -0.04384 & 0.04700 & -0.00820 & 0.00234 & -0.00279 & 0.001468 \\
$a_{17}^{1}\left(\mathrm{~cm}^{-19 / 2}\right)$ & $a_{19}^{1}\left(\mathrm{~cm}^{-21 / 2}\right)$ & $a_{21}^{1}\left(\mathrm{~cm}^{-23 / 2}\right)$ & $a_{23}^{1}\left(\mathrm{~cm}^{-25 / 2}\right)$ & $a_{25}^{1}\left(\mathrm{~cm}^{-27 / 2}\right)$ & $a_{27}^{1}\left(\mathrm{~cm}^{-29 / 2}\right)$ & $a_{29}^{1}\left(\mathrm{~cm}^{-31 / 2}\right)$ & $a_{31}^{1}\left(\mathrm{~cm}^{-33 / 2}\right)$ & $a_{33}^{1}\left(\mathrm{~cm}^{-35 / 2}\right)$ \\
-0.00126 & 0.01027 & -0.00068 & 0.00769 & -0.00041 & 0.00603 & -0.00027 & 0.00489 \\
$a_{35}^{1}\left(\mathrm{~cm}^{-37 / 2}\right)$ & $a_{37}^{1}\left(\mathrm{~cm}^{-39 / 2}\right)$ & $a_{39}^{1}\left(\mathrm{~cm}^{-41 / 2}\right)$ & $a_{41}^{1}\left(\mathrm{~cm}^{-43 / 2}\right)$ & $a_{43}^{1}\left(\mathrm{~cm}^{-45 / 2}\right)$ & $a_{45}^{1}\left(\mathrm{~cm}^{-47 / 2}\right)$ & $a_{47}^{1}\left(\mathrm{~cm}^{-49 / 2}\right)$ & $a_{49}^{1}\left(\mathrm{~cm}^{-51 / 2}\right)$ & $a_{51}^{1}\left(\mathrm{~cm}^{-53 / 2}\right)$ \\
0.00407 & -0.00013 & 0.00345 & -0.00009 & 0.00298 & -0.00007 & 0.00260 & -0.00005 & 0.00230 \\
\hline
\end{tabular}

Table 3. The coefficients of the Williams series expansion for the stress field in the vicinity of the crack tip $z=a$ (Fig. 6)

$(a=2 \mathrm{~cm}, b=0.5 \mathrm{~cm}, c=-0.5 \mathrm{~cm}, d=-1.5 \mathrm{~cm})$.

\begin{tabular}{lllllllll}
\hline$a_{1}^{1}\left(\mathrm{~cm}^{-3 / 2}\right)$ & $a_{2}^{1}\left(\mathrm{~cm}^{-2}\right)$ & $a_{3}^{1}\left(\mathrm{~cm}^{-5 / 2}\right)$ & $a_{5}^{1}\left(\mathrm{~cm}^{-7 / 2}\right)$ & $a_{7}^{1}\left(\mathrm{~cm}^{-9 / 2}\right)$ & $a_{9}^{1}\left(\mathrm{~cm}^{-11 / 2}\right)$ & $a_{11}^{1}\left(\mathrm{~cm}^{-13 / 2}\right)$ & $a_{13}^{1}\left(\mathrm{~cm}^{-15 / 2}\right)$ & $a_{15}^{1}\left(\mathrm{~cm}^{-17 / 2}\right)$ \\
\hline 0.62576 & -0.25000 & 0.20400 & -0.03470 & 0.01181 & -0.00499 & 0.00234 & -0.00117 & 0.00061 \\
$a_{17}^{1}\left(\mathrm{~cm}^{-19 / 2}\right)$ & $a_{19}^{1}\left(\mathrm{~cm}^{-21 / 2}\right)$ & $a_{21}^{1}\left(\mathrm{~cm}^{-23 / 2}\right)$ & $a_{23}^{1}\left(\mathrm{~cm}^{-25 / 2}\right)$ & $a_{25}^{1}\left(\mathrm{~cm}^{-27 / 2}\right)$ & $a_{27}^{1}\left(\mathrm{~cm}^{-29 / 2}\right)$ & $a_{29}^{1}\left(\mathrm{~cm}^{-31 / 2}\right)$ & $a_{31}^{1}\left(\mathrm{~cm}^{-33 / 2}\right)$ & $a_{33}^{1}\left(\mathrm{~cm}^{-35 / 2}\right)$ \\
-0.00033 & 0.00018 & -0.00010 & 0.00005 & -0.00003 & 0.00002 & -0.00001 & $0.733 \cdot 10^{-5}$ & $-0.442 \cdot 10^{-5}$
\end{tabular}




$$
(a=1.5 \mathrm{~cm}, b=0.5 \mathrm{~cm}, c=-0.5 \mathrm{~cm}, d=-1.5 \mathrm{~cm}) .
$$

\begin{tabular}{|c|c|c|c|c|c|c|c|c|}
\hline$a_{1}^{1}\left(\mathrm{~cm}^{-3 / 2}\right)$ & $a_{2}^{1}\left(c m^{-2}\right)$ & $a_{3}^{1}\left(\mathrm{~cm}^{-5 / 2}\right)$ & $a_{5}^{1}\left(\mathrm{~cm}^{-7 / 2}\right)$ & $a_{7}^{1}\left(\mathrm{~cm}^{-9 / 2}\right)$ & $a_{9}^{1}\left(\mathrm{~cm}^{-11 / 2}\right)$ & $a_{11}^{1}\left(\mathrm{~cm}^{-13 / 2}\right)$ & $a_{13}^{1}\left(\mathrm{~cm}^{-15 / 2}\right)$ & $a_{15}^{1}\left(\mathrm{~cm}^{-17 / 2}\right)$ \\
\hline 0.52397 & -0.25000 & 0.27937 & -0.04384 & 0.04700 & -0.00820 & 0.02343 & -0.00279 & 0.014687 \\
\hline$a_{17}^{1}\left(\mathrm{~cm}^{-19 / 2}\right)$ & $a_{19}^{1}\left(c^{-21 / 2}\right)$ & $a_{21}^{1}\left(\mathrm{~cm}^{-23 / 2}\right)$ & $a_{23}^{1}\left(c^{-25 / 2}\right)$ & $a_{25}^{1}\left(\mathrm{~cm}^{-27 / 2}\right)$ & $a_{27}^{1}\left(\mathrm{~cm}^{-29 / 2}\right)$ & $a_{29}^{1}\left(\mathrm{~cm}^{-31 / 2}\right)$ & $a_{31}^{1}\left(\mathrm{~cm}^{-33 / 2}\right)$ & $a_{33}^{1}\left(\mathrm{~cm}^{-35 / 2}\right)$ \\
\hline-0.00126 & 0.01027 & -0.000068 & 0.00769 & -0.00041 & 0.00603 & -0.00026 & 0.00489 & -0.00018 \\
\hline$a_{35}^{1}\left(\mathrm{~cm}^{-37 / 2}\right)$ & $a_{37}^{1}\left(\mathrm{~cm}^{-39 / 2}\right)$ & $a_{39}^{1}\left(\mathrm{~cm}^{-41 / 2}\right)$ & $a_{41}^{1}\left(\mathrm{~cm}^{-43 / 2}\right)$ & $a_{43}^{1}\left(\mathrm{~cm}^{-45 / 2}\right)$ & $a_{45}^{1}\left(\mathrm{~cm}^{-47 / 2}\right)$ & $a_{47}^{1}\left(\mathrm{~cm}^{-49 / 2}\right)$ & $a_{49}^{1}\left(\mathrm{~cm}^{-51 / 2}\right)$ & $a_{51}^{1}\left(\mathrm{~cm}^{-53 / 2}\right)$ \\
\hline 0.00407 & -0.00013 & 0.00345 & -0.00009 & 0.00298 & -0.00007 & 0.00260 & -0.00005 & 0.00230 \\
\hline
\end{tabular}

Table 5. The coefficients of the Williams series expansion for the stress field in the vicinity of the crack tip $z=d$

$$
(a=2.5 \mathrm{~cm}, b=0.5 \mathrm{~cm}, c=-0.5 \mathrm{~cm}, d=-1.5 \mathrm{~cm}) .
$$

\begin{tabular}{|c|c|c|c|c|c|c|c|c|}
\hline$a_{1}^{1}\left(c^{-3 / 2}\right)$ & $a_{2}^{1}\left(\mathrm{~cm}^{-2}\right)$ & $a_{3}^{1}\left(\mathrm{~cm}^{-5 / 2}\right)$ & $a_{5}^{1}\left(\mathrm{~cm}^{-7 / 2}\right)$ & $a_{7}^{1}\left(\mathrm{~cm}^{-9 / 2}\right)$ & $a_{9}^{1}\left(\mathrm{~cm}^{-11 / 2}\right)$ & $a_{11}^{1}\left(\mathrm{~cm}^{-13 / 2}\right)$ & $a_{13}^{1}\left(\mathrm{~cm}^{-15 / 2}\right)$ & $a_{15}^{1}\left(\mathrm{~cm}^{-17 / 2}\right)$ \\
\hline $\begin{array}{l}0.56275 \\
a_{17}^{1}\left(\mathrm{~cm}^{-19 / 2}\right)\end{array}$ & $\begin{array}{l}-0.25000 \\
a_{19}^{1}\left(\mathrm{~cm}^{-21 / 2}\right)\end{array}$ & $\begin{array}{l}0.31993 \\
a_{21}^{1}\left(\mathrm{~cm}^{-23 / 2}\right)\end{array}$ & $\begin{array}{l}-0.02563 \\
a_{23}^{1}\left(\mathrm{~cm}^{-25 / 2}\right)\end{array}$ & $\begin{array}{l}0.06089 \\
a_{25}^{1}\left(\mathrm{~cm}^{-27 / 2}\right)\end{array}$ & $\begin{array}{l}-0.00065 \\
a_{27}^{1}\left(\mathrm{~cm}^{-29 / 2}\right)\end{array}$ & $\begin{array}{l}0.02999 \\
a_{29}^{1}\left(\mathrm{~cm}^{-31 / 2}\right)\end{array}$ & $\begin{array}{l}0.00117 \\
a_{31}^{1}\left(\mathrm{~cm}^{-33 / 2}\right)\end{array}$ & $\begin{array}{l}0.01853 \\
a_{33}^{1}\left(\mathrm{~cm}^{-35 / 2}\right)\end{array}$ \\
\hline $\begin{array}{l}0.00122 \\
a_{35}^{1}\left(\mathrm{~cm}^{-37 / 2}\right)\end{array}$ & $\begin{array}{l}0.01286 \\
a_{37}^{1}\left(\mathrm{~cm}^{-39 / 2}\right)\end{array}$ & $\begin{array}{l}0.00106 \\
a_{39}^{1}\left(\mathrm{~cm}^{-41 / 2}\right)\end{array}$ & $\begin{array}{l}0.00958 \\
a_{41}^{1}\left(\mathrm{~cm}^{-43 / 2}\right)\end{array}$ & $\begin{array}{l}0.00901 \\
a_{43}^{1}\left(\mathrm{~cm}^{-45 / 2}\right)\end{array}$ & $\begin{array}{l}0.00749 \\
a_{45}^{1}\left(\mathrm{~cm}^{-47 / 2}\right)\end{array}$ & $\begin{array}{l}0.00766 \\
a_{47}^{1}\left(\mathrm{~cm}^{-49 / 2}\right)\end{array}$ & $\begin{array}{l}0.00060 \\
a_{49}^{1}\left(\mathrm{~cm}^{-51 / 2}\right)\end{array}$ & $\begin{array}{l}0.00065 \\
a_{51}^{1}\left(\mathrm{~cm}^{-53 / 2}\right)\end{array}$ \\
\hline 0.00504 & 0.00057 & 0.00027 & 0.00050 & 0.00368 & 0.00044 & 0.00321 & 0.00039 & 0.00284 \\
\hline
\end{tabular}

The teoretically reconstructed isochromatic fringes calculated by the use of the Williams series expansion with the coefficients presented in Tables 1-5 are shown in Fig. 7. Here blue points are experimentally chosen points and the red lines are theoretically obtained contours of the diffferences of the principal stresses. One can see good consistency the theoretical results with the experimental points. Our analysis shows that the higher order terms of the Williams series expansion play significant role in the description of the stress field in the vicinity of the crack tip.

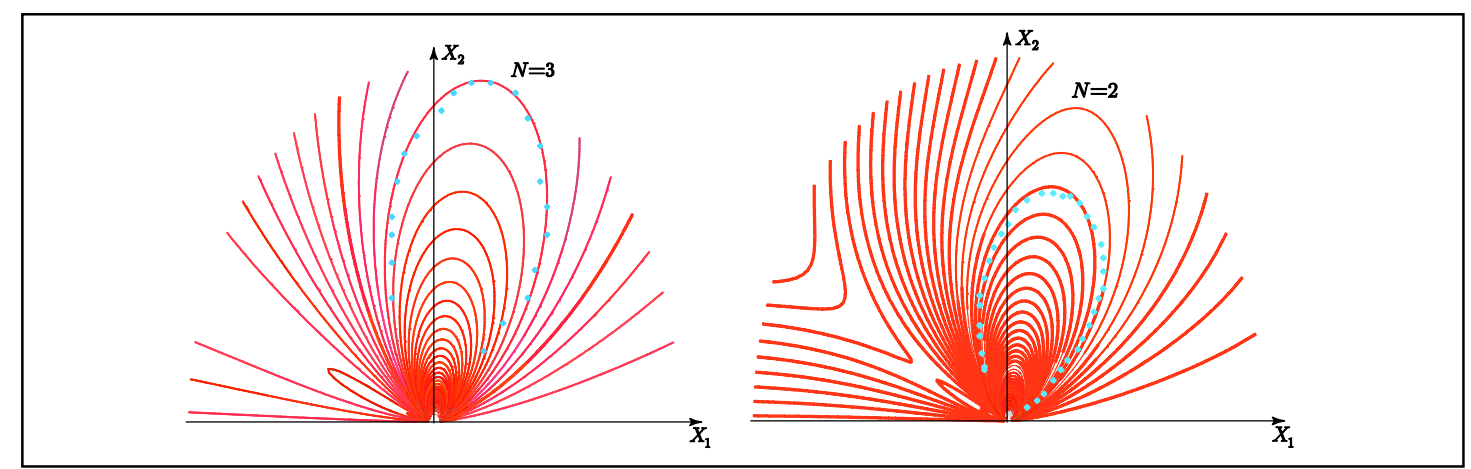

Fig. 7. Experimental points and theoretically constructed isochromatic fringe patterns in the vicinity of the crack tip in the plate with two collinear cracks of different lenght.

A bitmap image can be constructed by calculating the intensity numerically at each point in a large array of regularly spaced points and observing the resulting pattern. An example is shown in Fig. 10 for dark-field isochromatics in multi-parameter stress field based on Eq. 1.
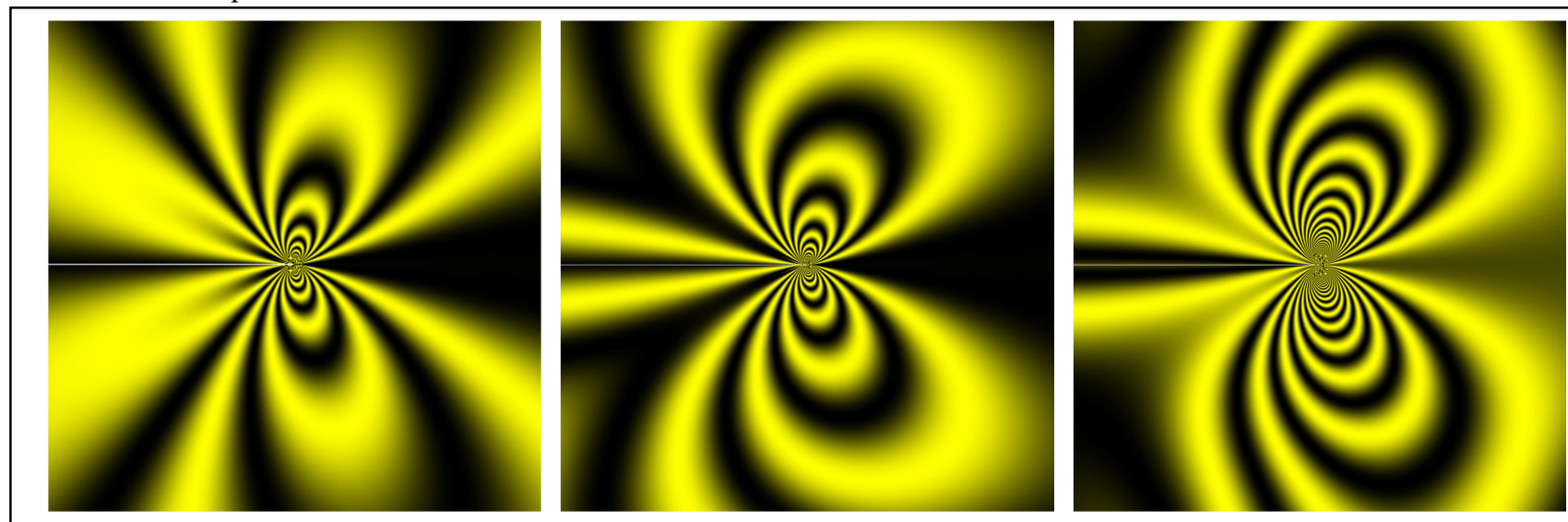

Fig. 8. Simulated multi-parameter stress field in the vicinity of the crack tip with 2,5 and 9 terms of the Williams seies expansion of the stress field.

The contours of the Mises equivalent stress in the vicinity of the Mode I crack tip and Mode II crack tip are shown in Fig. 9 and Fig. 10 respectively. From Fig. 9 (left) one can see the contours of the Mises equivalent stress in the vicinity of the Mode I crack tip obtained by the leading order term of the Williams series expansion shown by red lines and the exact solution of the 
problem and multi-parameter description of the stress field [16] shown by blue lines. One can see that the asymptotic solution and the exact solution conicide only in the vicinity of the crack tip at very small distances from the crack tip. Increasing the number of terms of the Williams expansion results in extension of the domain where the Williams asymptotic expansion is valid. Finally, Figure 9 (right) shows the contours of the Mises equivalent stress obtained by the Williams expansion keeping 100 terms (red lines). The red lines coincide completely with the exact solution for the infinite elastic medium [16]. The similar results have been obtained for mode II (Fig. 10) and mixed mode crack problems.
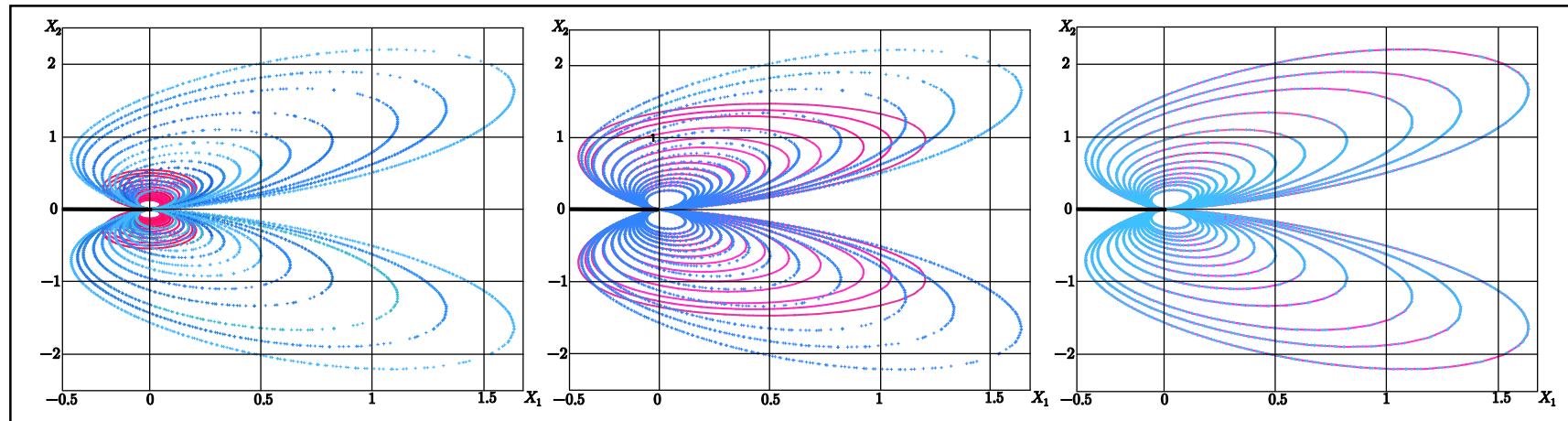

Fig. 9. Influence of the higher order terms of the Williams asymptotic expansion.
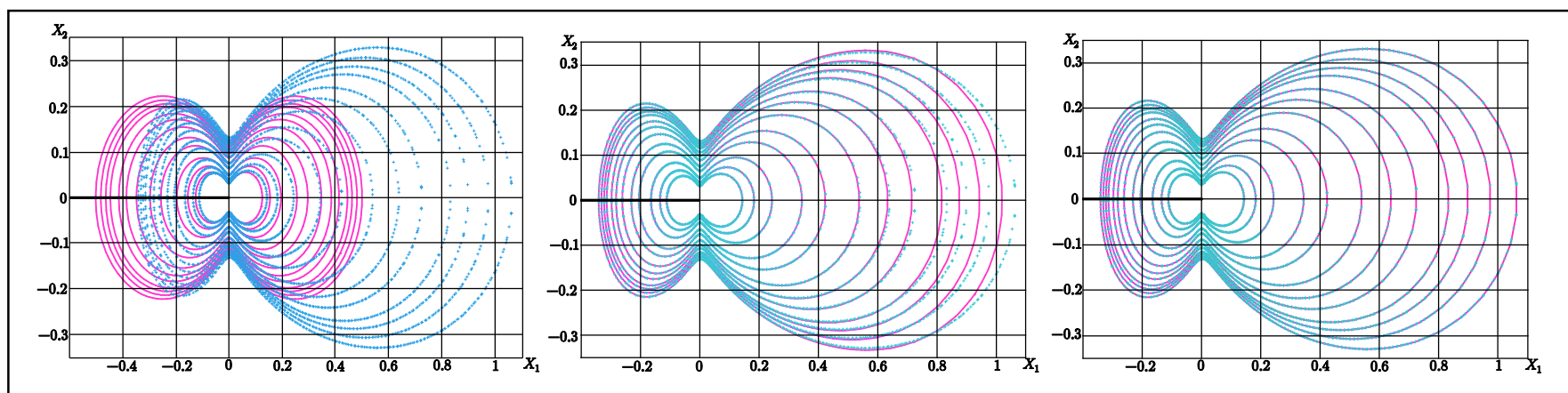

Fig. 10. Influence of the higher order terms of the Williams asymptotic expansion.

The first coefficients of the Williams series expansions $a_{1}^{1}$ and $a_{1}^{2}$ were compared with the theoretical results known in literature for stress intensity factors for an infinite plane medium with two collinear cracks [35]:

$$
\begin{aligned}
& \left\{\begin{array}{l}
K_{I} \\
K_{I I}
\end{array}\right\}_{b}=\left\{\begin{array}{l}
\sigma_{22}^{\infty} \\
\sigma_{12}^{\infty}
\end{array}\right\} \sqrt{\pi(a-b) / 2} \frac{1}{\sqrt{1-\alpha_{b}}}\left\{1-\frac{1}{\alpha_{a}}\left[1-\frac{E(k)}{K(k)}\right]\right\}, \\
& \alpha_{a}=(a-b) / l_{A}, \quad \alpha_{b}=(c-d) / l_{B}, \quad k=\sqrt{\alpha_{a} \alpha_{b}} .
\end{aligned}
$$

The experimentally obtained coefficients and the theoretical results are in a good agreement. The exact expressions for the coefficients of the higher order terms in the Williams series expansions known for the plate with two collinear cracks have been used either for checking the results of the program developed. The accuracy of the method has been tested for several configuration subjected to pure Mode I and Mixed-Mode loadings.

\section{Results and Discussion}

In this research, photoelasticity is employed to assess coefficients of the complete Williams series expansion of the linear elastic stress field in the vicinity of the crack tip for a wide class of experimental specimens subject to mixed mode loading: plates with two collinear cracks of equal and different lengths under tensile loading and mixed mode loading. The study has showed that the coefficients of higher order terms can play an important role in fracture process in notched and cracked structures. The approach developed allows us to construct all the higher order terms in the asymptotic expansions in order to better approximate stress field. We use the plates with two collinear cracks under mixed mode loading to construct the complete multi-parameter asymptotic expansion of the stress field in the vicinity of the crack tip.

By means of photoelasticity the distribution of the isochromatic fridge patterns and the stress field near the crack tip based on the complete Williams asymptotic expansion for various classes of the experimental specimens under mixed mode loading are obtained. In the present contribution fracture mechanics problems regarding the study of the stress and displacement fields around a crack tip under mixed mode loading are discussed in the framework of photoelastic techniques and an overdeterministic method for calculation of the coefficients of crack tip asymptotic field. The comparison of the experimental results and the calculations performed with finite element analysis has shown the importance and significant advantages of photoelastic observations for the multiparametric description of the stress field in the neighborhood of the crack tip. 


\section{Conclusion}

The study is aimed at experimental and computational determination of the coefficients in the crack tip stress asymptotic expansions for a wide class of specimens under mixed mode loading conditions. In the paper multiparametric presentation of the stress filed near the crack tips for a wide class of specimens is obtained. Theoretical, experimental and computational results obtained in this research show that the isochromatic fringes in the vicinity of the crack tip are described more accurately when we consider the complete Williams asymptotic expansion of the stress field and we have to keep the higher order stress terms in the asymptotic expansion since the contribution of the higher order stress terms (besides the stress intensity factors and the Tstress) is not negligible in the crack tip stress field. The example problems revealed the advantage of using a multi-parameter solution in terms of collecting data from a larger zone. A good correlation was observed between the experimental results and the numerical results obtained from finite element analysis.

\section{References}

[1] Asundi AK. Matlab for Photomechanics. Elsevier, London, 2002.

[2] Aytollahi MR, Mirsayar MM, Dehghany M. Experimental determination of stress field parameters in bi-material notches using photoelasticity. Materials and Design 2011; 32: 4901-4908.

[3] Saravani M, Azizi M. The investigation of crack's parameters on the V-notch using photoelasticity method. International Journal of Mechanical, Aerospace, Industrial, Mechatronic and Manufacturing Engineering 2011; 5(1): 152-157.

[4] Guagliano M, Sangirardi M, Sciuccati A, Zakeri M. Multiparameter Analysis of the stress field around a crack tip. Procedia Engineering 2011; 10: 29312936.

[5] Surendra KVN, Simha KRY. Design and analysis of novel compression fracture specimen with constant form factor: Edge cracked semicircular disk (ECSD). Engineering Fracture Mechanics 2013; 102: 235-248.

[6] Lei Z, Okunev AO, Zhu C, Verozubova GA, Ma T. Photoelasticity method for study of structural imperfection of ZnGeP2 crystal. Journal of Crystal Growth. 2016; 450: 34-38.

[7] Magalhaes junior PAA, Magalhaes CA, Magalhaes ALMA. Computational methods of phase shifting to stress measurement with photoealsticity using plane polariscope. Optik 2017; 130: 213-226.

[8] Ramesh K, Ramakrishnnan V. Digital photoelasticity of glass: A comprehensive review 2016; 87: 59-74.

[9] Stepanova LV, Roslyakov PS. Complete asymptotic expansion m. williams near the crack tips of collinear cracks of equal lengths in an infinite plane medium. PNRPU Mechanics Bulletin 2015; 4: 188-225.

[10] Stepanova LV, Igonin SA. Asymptotics of the near-crack-tip stress field of a growing fatigue crack in damaged materials: Numerical experiment and analytical solution. Numerical Analysis and Applications 2015; 8(2): 168-181.

[11] Patil P, Vyasarayani CP, Ramji M. Linear least squares approach for evaluating crack tip fracture parameters using isochromatic and isoclinic data from digital photoelasticity. Optic and Lasers in Engineering 2017; 93: 182-194.

[12 ] Ayatolloahi MR, Dehghany M, Mirsayar MM. A comprehensive study for mode I sharp V-notches. European Journal of Mechanics A/Solids 2013; 37: $216-230$.

[13] Harilal R, Vyasarayani CP, Ramji M. A linear least squares approach for evaluation of crack tip stress field parameters using DIC. Optic and lasers in Engineering 2015; 75: 95-102.

[14] Ayatollahi MR, Nejati M. Experimental evaluation of stress field aroun the sharp notches using photoelasticity. Materials and Design 2011; 32: 561-569.

[15] Ayatollahi MR, Moazzami M. Digital image correlation method for calculating coefficients of Williams expansion in compact tension specimen. Optic and Lasers in Engineering 2017; 90: 26-33.

[16] Stepanova L, Roslyakov P. Multi-parameter description of the crack-tip stress field: Analytic determination of coefficients of crack-tip stress expansions in the vicinity of the crack tips of two finite cracks in an infinite plane medium. International Journal of Solids and Structures 2016; 100-101: 11-28.

[17] Ramesh K, Gupta S, Kelkar AA. Evaluation of stress field parameters in fracture mechanics by photoelasticity - revisited. Engineering fracture mechanics 1997; 56(1): 25-45.

[18] Pisarev VS, Matvienko YG, Eleonsky SI, Odintsev IN. Combining the crack compliance method and speckle interferomentry data for determination of stress intensity factors and T-stressю Engineering fracture mechanics 2017; 179: 348-374.

[19] Ayatollahi MR, Zakeri M. An improved definition for mode I and mode II crack problems. Engineering fracture mechanics 2017; 175: 235-246.

[20] Sestakova L. Using the multi-parameter fracture mechanics fore accuraye description of stress/displcement crack-tip fields. Key Engineering Materials 2014; 586: 237-240.

[21] Hello G, Tahar M-B, Roeland J-M. Analytical determination of coefficients in crack-tip stress expansions for a finite crack in an infinite plane medium. International Journal of Solids and Structurtes 2012; 49: 556-566.

[22] Vesely V, Sobek J, Frantik P, Seitl S. Multi-parameter approximation of the stress field in a cracked body in the more distant surroundings of the crack tip. International Journal of Fatigue 2016; 89: 20-35.

[23] Stepanova LV, Yakovleva EM. Mixed-mode loading of the cracked plate under plane stress conditions. PNRPU Mechanics Bulletin 2014; 3: 129-162.

[24] Malikova L, Vesely V. Influence of the elastic mismatch on crack propagation in a silicate-based composite. Theoretical and Applied Fracture Mechanics, 2017.

[25] Malikova L, Vesely V, Seitl S. Estimation of the crack propagation direction in a mixed-mode geometry via multi-parameter fracture criteria. Frattura ed Integrita Strutturale 2015; 33: 25-32.

[26] Akbardoost J, Rastin A. Comprehensive date for calculating the higher order terms of crack tip stress field in disk type specimens under mixed-mode loading. Theoretical and Applied Fracture Mechanics 2015; 76: 75-90.

[27] Hello G, Tahar M-B. On the exactness of truncated crack-tip stress expansions. Procedia Materials Science 2014; 3: 750-755.

[28] Holyns'kyi IS. Influence of errors of determination of stresses near a crack tip on the accuracy of computation of the coefficients of the Williams series under Mode II loading. Materials Science 2013; 48(4): 438-443.

[30] Stepanova LV, Adylina EM. Stress-strain state in the vicinity of a crack tip under mixed loading. Journal of Applied Mechanics and Technical Physics 2014; 55(5): 885-895.

[31] Lychak O, Holyns'kyi IS. Evaluation of random errors in Williams' series coefficients obtained with digital image correlation. Measurement Science and Technology 2016; 27(3): 035203.

[32] Stepanova LV, Igonin SA. Rabotnov damageparameter and description of delayed fracture: Results, current status, application to fracture mechanics, and prospects. Journal of Applied Mechanics and Technical Physics 2015; 56(2): 282-292.

[33] Berto F, Lazzarin P. Multiparametric full-field representations of the in-plane stress fields ahead of cracked components under mixed mode loading. International Journal of Fatigue 2013; 46: 16-26.

[34] Stepanova LV, Yakovleva EM. Asymptotic stress field in the vicinity of a mixed-mode crack under plane stress conditions for a power-law hardening material. Journal of Mechanics of Materials and Structures 2015; 10(3): 367-393.

[35] Tada H, Paris PC, Irwin GR. The stress analysis of cracks. Handbook, ASME Press, New York, 2000. 